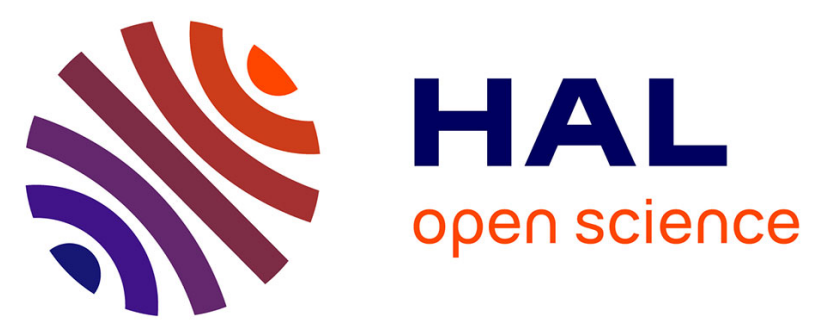

\title{
Real-time computer modeling in prevention of foot pressure ulcer using patient-specific finite element model and model order reduction techniques
}

S. Niroomandi, A. Perrier, M. Bucki, Yohan Payan

\section{- To cite this version:}

S. Niroomandi, A. Perrier, M. Bucki, Yohan Payan. Real-time computer modeling in prevention of foot pressure ulcer using patient-specific finite element model and model order reduction techniques. Amit Gefen. Innovations and Emerging Technologies in Wound Care, Elsevier, pp.87-102, 2020, 9780-12-815028-3. 10.1016/B978-0-12-815028-3.00005-5 . hal-02356288

\section{HAL Id: hal-02356288 \\ https://hal.science/hal-02356288}

Submitted on 8 Nov 2019

HAL is a multi-disciplinary open access archive for the deposit and dissemination of scientific research documents, whether they are published or not. The documents may come from teaching and research institutions in France or abroad, or from public or private research centers.
L'archive ouverte pluridisciplinaire HAL, est destinée au dépôt et à la diffusion de documents scientifiques de niveau recherche, publiés ou non, émanant des établissements d'enseignement et de recherche français ou étrangers, des laboratoires publics ou privés. 


\title{
Real-time computer modeling in prevention of foot pressure ulcer using patient-specific finite element model and model order reduction techniques
}

\author{
Niroomandi S., Perrier A., Bucki M. and Payan Y.
}

\section{Introduction}

This chapter summarizes some technologies that could be used to help with prevention of diabetic foot pressure ulcer. The latest work from the authors is also presented. The first component is a fully wireless, customizable and washable smart sock to monitor the pressure applied to the foot. The second component is a patient-specific Finite Element model of the foot including soft tissues and bony prominences that is specifically built and used to compute the internal strains and stresses from the pressure data provided by the smart sock sensors. To simulate such complex model, the ArtiSynth platform (artisynth.org) was used. Although ArtiSynth is powerful in combining rigid bodies and soft tissues, simulation time is in the order of hours which is not suitable for a monitoring device. To overcome this issue we could use a reduced order model to decrease computation time to less than a second and thus make the simulations run in real-time. In the following sections foot pressure ulcer and associated complications are briefly reviewed while the smart diabetic socks are described. Then a patient-specific foot model from the authors is presented. Afterwards, main ideas of model order reduction techniques that are used namely Proper Orthogonal Decomposition (POD) and POD with Interpolation (PODI) are reviewed. After the reduced order model is constructed a displacement field for evaluation points was simulated in a time frame of about 0.1s. Despite such short computation time the results of the reduced order model show good accuracy in comparison to the full Artisynth solution and the maximum relative error norm was $2.6 \%$ which seems acceptable from a clinical point of view. This technique could equally be extended to use an online version of the foot model by applying pressure values measured by the smart sock. 


\section{Foot ulcer and smart diabetic socks}

Foot ulcers are mostly the consequence of a trauma or exessive pressures associated to diabetes and resulting from repetitive high stresses and strains, ill-fitting footwear or sometimes an external object inside the shoe. Diabetic peripheral neuropathy and angiopathy are chronic complications of diabetes affecting the lower limbs. When both complications are associated to an external trivial trauma, they determine the progression of the pathology from a non-ulcerated condition of a foot at risk, to an acute syndrome characterized by the classic diabetic foot ulcer, followed by a chronic condition in the post-ulcerative phase, which can lead to amputation [Shaw and Boulton, 1997]. Healthy individuals with undamaged somatosensory function would experience pain and avoid the offending pressures if such external trauma occurred. On the contrary, in a person who suffers from diabetic neuropathy there is no warning of excessive pressure or tissue damage which could lead to skin breakdown or ulceration. Previous studies suggested that an efficient care could reduce foot ulcer [Boulton et al., 2005]. This monitoring strategy is largely based on renewed vigilance of the diabetic patient which mainly consists in making a daily inspection of his/her feet. Unfortunately, studies show that patient vigilance decreases over time and complications which appear after some time are directly correlated with the increasing lack of vigilance. In the case of diabetic patients, it is precisely when the first foot ulcers appear that serious complications begin. This is mainly due to recurrent disease in the diabetic angiopathy, which severely limits the healing process. It becomes therefore essential to propose new technologies and services dedicated to the monitoring and assistance of the diabetic patient to help maintain his/her alertness and by extension his/her autonomy in society. The external trauma that causes tissue breakdown could be intrinsic, such as repetitive stresses from high pressure and/or callus, or extrinsic such as from ill-fitting footwear rubbing on the skin or an object inside the shoe. It hence appears very suitable to be able (1) to monitor the external pressure applied all around the foot and (2) if possible, to estimate the corresponding internal stresses and strains.

In order to monitor the external pressures a fully wireless customizable and washable smart sock was designed in a collaboration between two research laboratories (TIMC-IMAG and AGIM), three companies (TexiSense, IDS and IFTH) and a clinical center (Centre de l'Arche) in France [Perrier et al., 2014].This smart sock is made of three main components: a $100 \%$ textile sock that collects the foot external pressures, a central unit, and an external device that receives the wireless information sent by the central unit and estimates the risks for pressure ulcer. A schematic of this system and 
details of the sock and its embedded sensors are shown in Fig. 1 and Fig. 2 respectively.
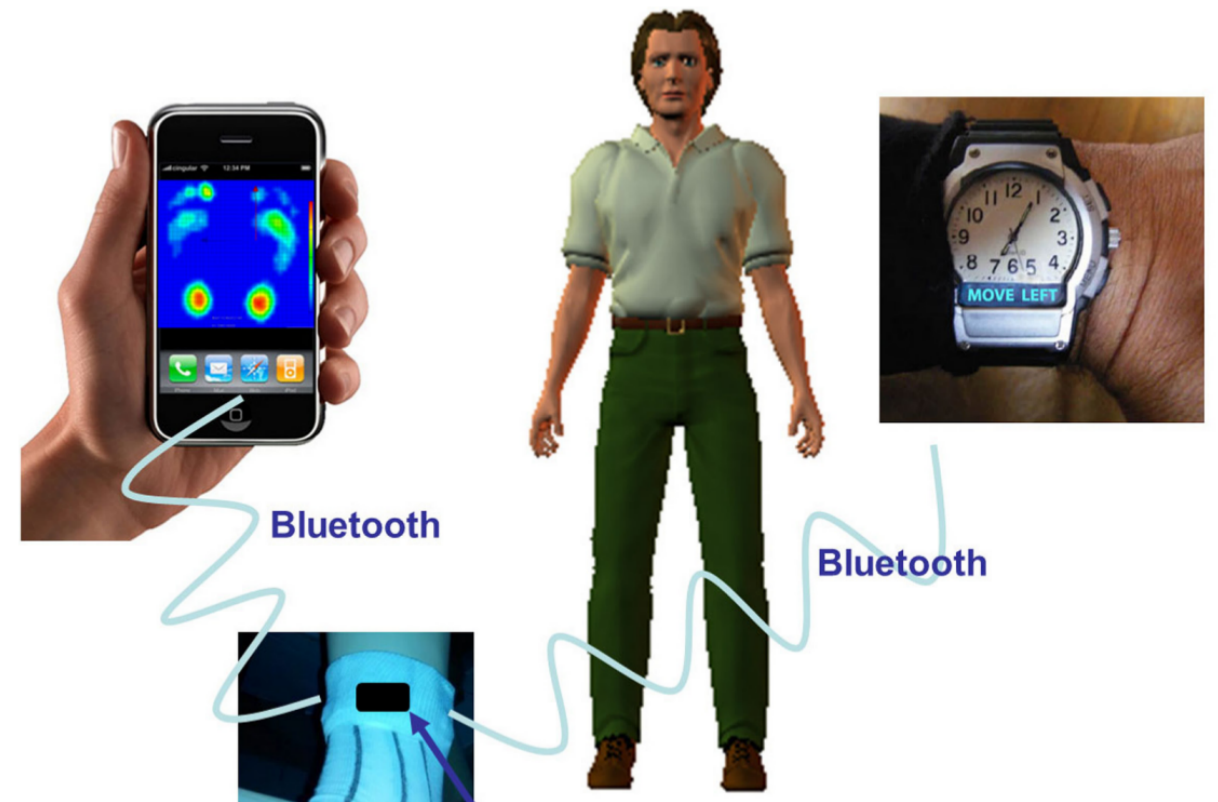

\section{The Smart Diabetic Socks}
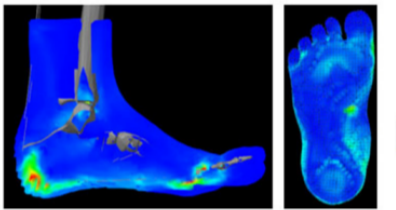

Central Unit

Finite Element

Model

Figure 1: This smart sock is made of three main components: a 100\% textile sock that collects the foot external pressures, a central unit, and an external device that receives the wireless information sent by the central unit and estimates the risks for pressure ulcer. From [Perrier et al., 2014]

It is now well established that measuring pressures at the skin surface is not sufficient to prevent the most dangerous foot ulcers that start in deep tissues and progress outward rapidly, causing substantial subcutaneous damage underneath intact skin [Gefen, 2003, Atlas et al., 2009]. Indeed, such surface measurements are not sufficient to predict ulcer formation caused by internal tissue loading [Linder-Ganz et al., 2008, Loerakker et al., 2011]. For example, a very similar pressure distribution could be observed under the heel of a thin person with blunt calcaneus bone and of a heavy diabetic person with sharp calcaneus bone, whereas in that case, the diabetic person has obviously much higher risks for foot pressure ulcers. The likelihood of a pressure ulcer 

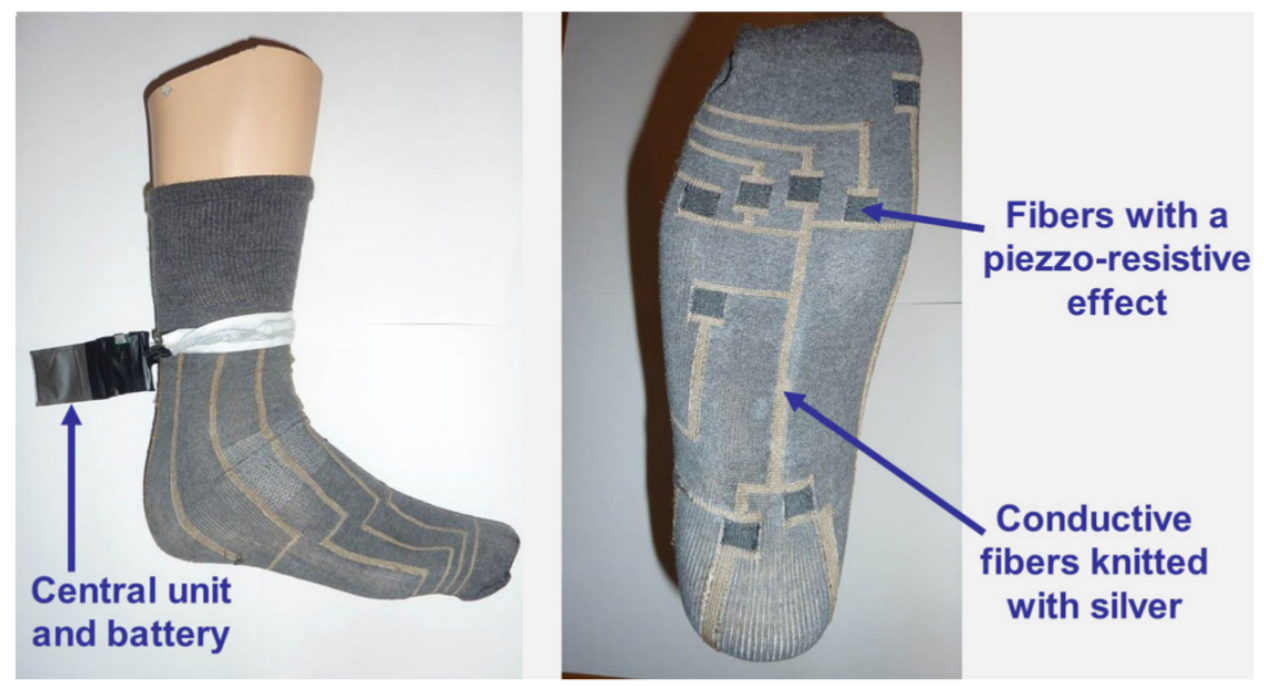

Figure 2: The sock knitted with fibers coated with silver (current conduction) and with fibers having a piezo-resistive effect. The central unit (left) is connected to the silver-coated fibers and to a soft battery. From [Perrier et al., 2014]

forming is therefore highly patient-specific since it depends on the anatomical properties of the patient foot such as the bones curvatures (calcaneus, metatarsal heads, toes) as well as the thicknesses of the soft tissues layers (skin, fat and muscles). Since measuring in vivo and in an embedded way the internal strains of the foot tissues is not possible, we have proposed to quantitatively estimate the internal stresses and strains from the measured external pressures. To achieve this goal, a patient-specific Finite Element model of the foot including soft tissues and bony prominences is specifically built and used to compute the internal strains and stresses (Fig. 3).

\section{A patient-specific foot model}

A detailed biomechanical model of the foot has been developed in [Perrier et al., 2015]. For this, a high-resolution CT volume acquired on the unloaded right foot of a volunteer was used to segment the 28 bones and reconstruct their 3D shapes. Rigid body contact constraints were implemented in the Artisynth framework to model joint interactions. All 33 joint motions were further constrained by 210 ligaments modeled by cables and inserted on the bones using CT images of the subject. Such contacts between bones and ligaments attached to the bones were thus used to guide the foot kinemat- 


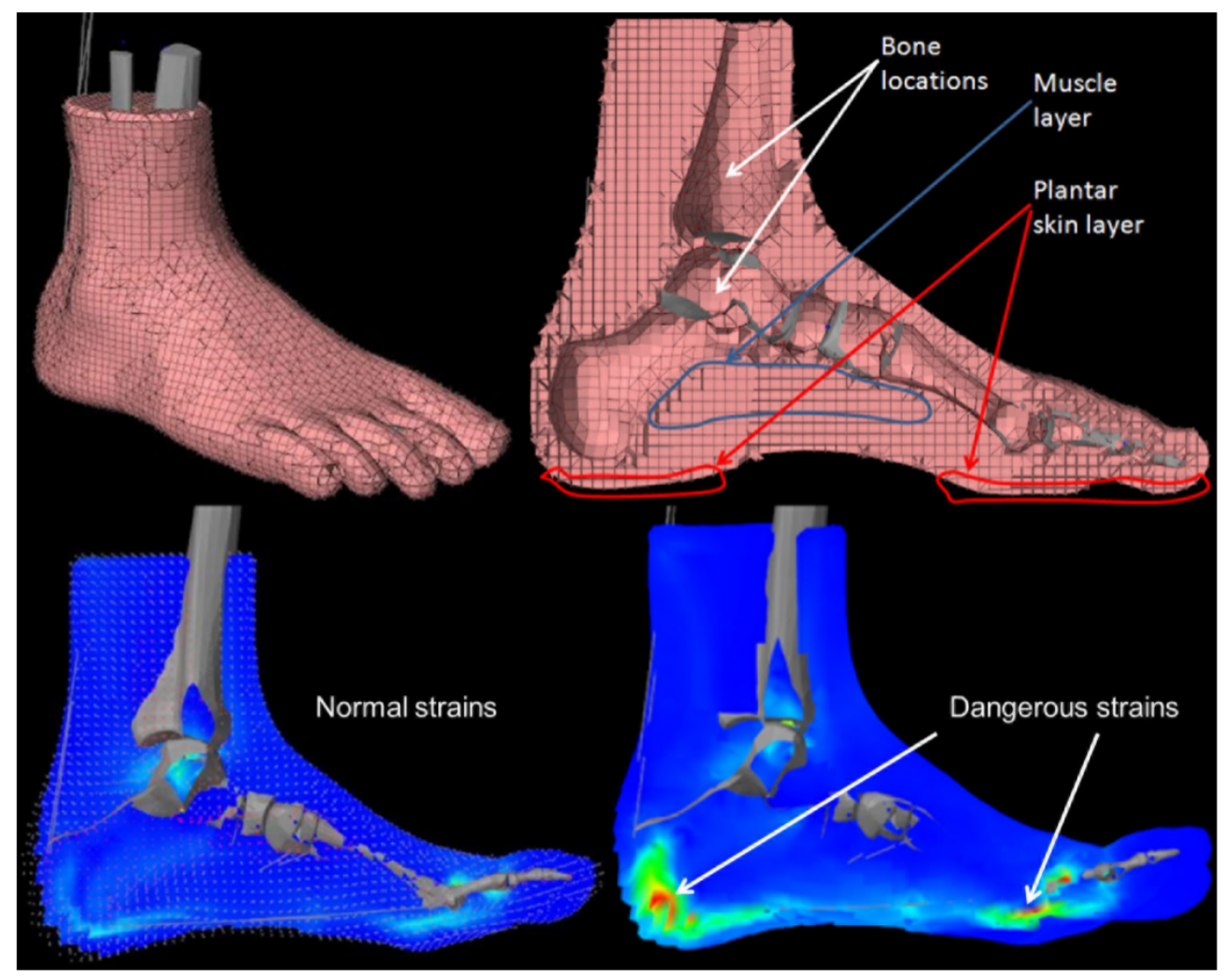

Figure 3: Finite Element model of the foot: a 3D mesh (top) is used to compute internal strains (bottom). If strains values become too high (as it is illustrated in the lower right panel), an alert is launched. From [Perrier et al., 2014]

ics. The Aponeurosis was modeled using five ligaments linked by transverse structures. Finally, 15 Hills model muscles were positioned according to their anatomical course and can be independently activated. Soft tissues were modeled by a Finite Element (FE) mesh comprising three sub-domains representing skin, fat and muscle tissues. The bones were modeled as rigid bodies to accelerate the simulation. The behavior of the bony rigid bodies coupled with finite element models of the soft tissue domains was computed using a semi-implicit integration scheme in the ArtiSynth open source framework [Lloyd et al., 2012]. In order to increase the realism from a physiological and a dynamic point of view, the masses of the different structures were estimated using the tables from [de Leva, 1996], which yielded a foot mass of $1.37 \%$ of the subject mass. The density and mass of the bones and soft tissues are shown in Table 1.

The muscles and part of the ligament insertions were localized within the 
Table 1: The density and mass of the bones and soft tissues

\begin{tabular}{lll} 
& Density $\left(\mathrm{kg} / \mathrm{m}^{3}\right)$ & Mass $(\mathrm{kg})$ \\
\hline Bones & 1850 & 0.433 \\
Soft tissues & 1200 & 1.13 \\
\hline
\end{tabular}

medical images, and the other part of the ligament insertions by resorting to the literature, Fig. 4.

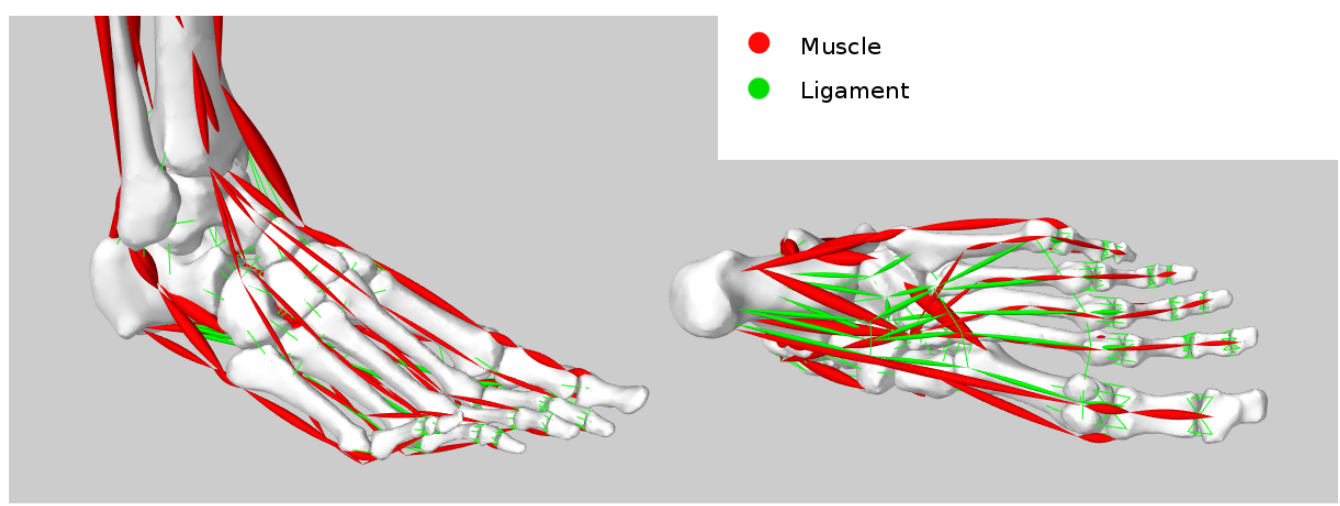

Figure 4: The musculoskeletal model with 28 bones, 15 activated muscles (in red) and 210 ligaments (in green). The insertions of those structures have been determined from the MRI scan and from literature.

The soft tissue FE mesh was generated using a meshing tool provided by the Texisense Company. In this application, modeling domains are defined by the 3D surfaces recovered from medical image segmentation. The meshing algorithm tends to maximize the number of hexahedrons in the generated mesh to avoid the locking effect inherent to linear tetrahedrons in nearlyincompressible materials. The final mesh is composed of 23298 elements and 13087 nodes. It is composed of four domains: skin, heel fat pad, other fatty tissues, and muscles (see Fig. 5). Since bones are modeled as rigid bodies, they do not need to be discretized.

In order to replicate a nonlinear hyperelastic behavior an isotropic neoHookean material is assumed to describe the behavior of the soft tissues. This material behavior is characterized by the strain density energy function given by

$$
\Psi=C_{10}\left(I_{1}-3\right)+(J-1)^{2} / D
$$

where $I_{1}$ is the first invariant of the right Cauchy-Green deformation tensor, $C_{10}$ is a material parameter, $\mathrm{J}$ is the determinant of the deformation gradient, 


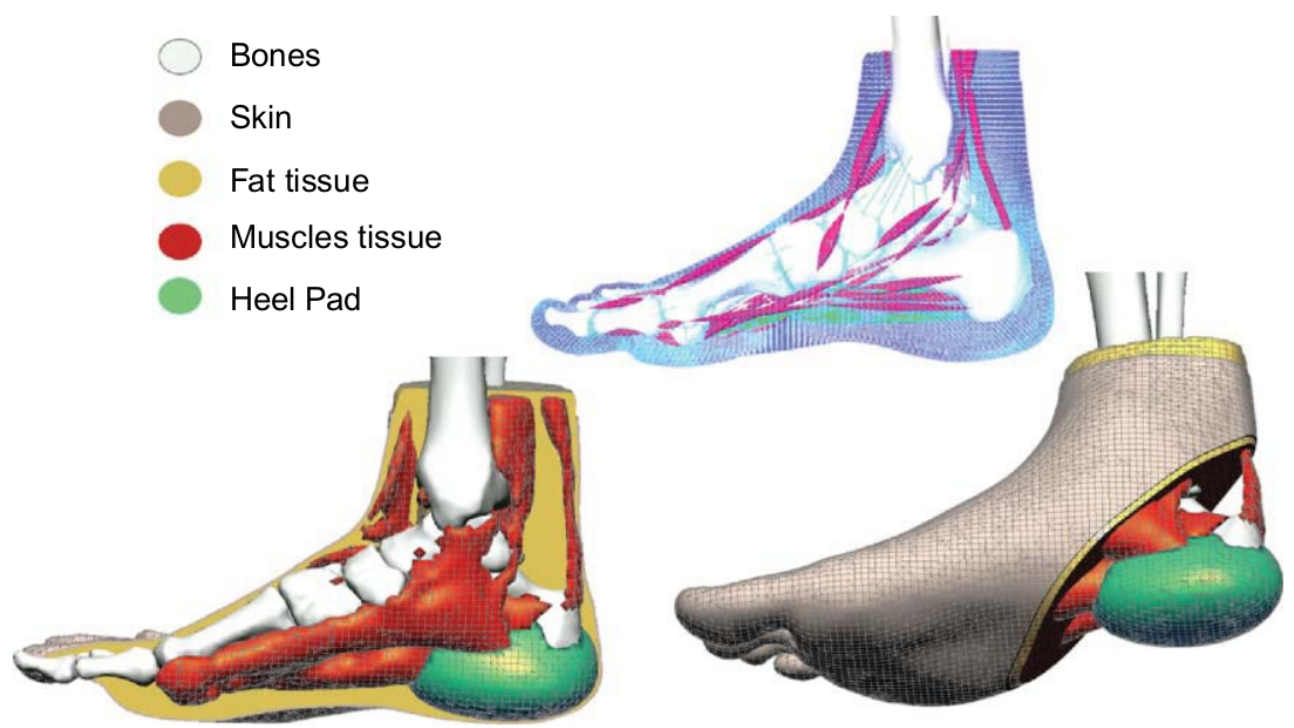

Figure 5: The complete biomechanical foot model with bony structures represented as rigid bodies, and soft tissues modeled as a finite element mesh. Soft tissues are divided in four layers: skin (in beige color), heel fat pad (in green), other fatty tissue (in yellow), and muscles (in red). From [Perrier et al., 2017]

and $\mathrm{D}$ is a material incompressibility parameter. The material properties of each soft tissue layer (or domain) are shown in Table 2 [Luboz et al., 2015]. More details about this model can be found in [Perrier et al., 2015, 2017].

Table 2: Material properties of the neo-Hookean model for each soft tissue

\begin{tabular}{lcc} 
& $C_{10}$ & Poisson's ratio \\
\hline Skin & 33.3 & 0.485 \\
Heel fat pad & 16.6 & 0.499 \\
Other fat tissues & 5.0 & 0.490 \\
Muscles & 10.0 & 0.495 \\
\hline
\end{tabular}

\section{Model order reduction}

The technique we used in this work goes by many names as it was employed in different branches of science and engineering among which Karhunen-Loève (KL) decomposition, proper orthogonal decomposition (POD) or singular 
value decomposition (SVD) can be mentioned [Karhunen, 1946, Sirovich, 1987, Krysl et al., Liang et al., 2002, Pinnau, 2008]. POD was successfully applied to real-time simulation of soft tissue [Niroomandi et al., 2008, 2010, Cueto and Chinesta, 2014]. In this work a variation of that method called POD with Interpolation (PODI) was used to construct a reduced order model with a parameter which is the muscle activation. Such a model makes it possible to have a fast computation of the field of interest for any value of the parameter [Ly and Tran, 2001, Epureanu et al., 2001].

\subsection{Proper Orthogonal Decomposition}

In this technique we assume that the evolution of a certain field $u(\boldsymbol{x}, t)$ is known for example displacements. In practical applications (assuming that some numerical simulations were performed off-line, for instance), this field is expressed in a discrete form which is known at the nodes $i$ of a spatial mesh at time instance $t^{m}$. Thus, we consider that $u\left(\boldsymbol{x}_{i}, t^{m}\right) \equiv u_{i}^{m}$ are known. We can also write $\boldsymbol{u}^{m}$ for the vector containing the nodal displacements at time instance $t^{m}$. The main idea of Karhunen-Loève (KL) decomposition is to obtain the most typical or characteristic structure $\phi(\boldsymbol{x})$ among these $u^{m}(\boldsymbol{x}), \forall m$. Here $\phi$ is a scalar field defined at all the nodes of the FE mesh. The characteristic structure is defined in the KL approach as the function that maximizes:

$$
\frac{\sum_{m=1}^{M}\left[\sum_{i=1}^{N} \phi\left(\boldsymbol{x}_{i}\right) u^{m}\left(\boldsymbol{x}_{i}\right)\right]^{2}}{\sum_{i=1}^{N}\left(\phi\left(\boldsymbol{x}_{i}\right)\right)^{2}}
$$

where $N$ represents the number of nodes of the complete model and $M$ the number of computed time steps. The maximization is equivalent to finding for any direction $\tilde{\phi}$ the zero of the Gâteaux derivative of the right hand side in the above equation, which leads to

$$
\sum_{m=1}^{M}\left[\left(\sum_{i=1}^{N} \tilde{\phi}\left(\boldsymbol{x}_{i}\right) u^{m}\left(\boldsymbol{x}_{i}\right)\right)\left(\sum_{j=1}^{N} \phi\left(\boldsymbol{x}_{j}\right) u^{m}\left(\boldsymbol{x}_{j}\right)\right)\right]=\alpha \sum_{i=1}^{N} \tilde{\phi}\left(\boldsymbol{x}_{i}\right) \phi\left(\boldsymbol{x}_{i}\right) ; \quad \forall \tilde{\phi}
$$

which can be rewritten in the form

$$
\sum_{i=1}^{N}\left\{\sum_{j=1}^{N}\left[\sum_{m=1}^{M} u^{m}\left(\boldsymbol{x}_{i}\right) u^{m}\left(\boldsymbol{x}_{j}\right) \phi\left(\boldsymbol{x}_{j}\right)\right] \tilde{\phi}\left(\boldsymbol{x}_{i}\right)\right\}=\alpha \sum_{i=1}^{N} \tilde{\phi}\left(\boldsymbol{x}_{i}\right) \phi\left(\boldsymbol{x}_{i}\right) ; \forall \tilde{\phi}
$$

Defining the vector $\boldsymbol{\phi}$ such that its $i$-th component is $\phi\left(\boldsymbol{x}_{i}\right)$, Eq. (4) takes the following matrix form

$$
\tilde{\phi}^{T} \boldsymbol{c} \phi=\alpha \tilde{\phi}^{T} \phi ; \forall \tilde{\phi} \Rightarrow \boldsymbol{c} \phi=\alpha \phi
$$


where the two-point correlation matrix is given by

$$
c_{i j}=\sum_{m=1}^{M} u^{m}\left(\boldsymbol{x}_{i}\right) u^{m}\left(\boldsymbol{x}_{j}\right) \Leftrightarrow \boldsymbol{c}=\sum_{m=1}^{M} \boldsymbol{u}^{m}\left(\boldsymbol{u}^{m}\right)^{T}
$$

which is symmetric and positive definite. If we define the matrix $\boldsymbol{Q}$ containing the discrete field history:

$$
\boldsymbol{Q}=\left(\begin{array}{llll}
u_{1}^{1} & u_{1}^{2} & \cdots & u_{1}^{M} \\
u_{2}^{1} & u_{2}^{2} & \cdots & u_{2}^{M} \\
\vdots & \vdots & \ddots & \vdots \\
u_{N}^{1} & u_{N}^{2} & \cdots & u_{N}^{M}
\end{array}\right)
$$

then it is easy to verify that the matrix $\boldsymbol{c}$ in Eq. (5) results

$$
\boldsymbol{c}=\boldsymbol{Q} \boldsymbol{Q}^{T}
$$

If some simulations were carried out, we can determine $\boldsymbol{u}_{i}^{m}, \forall i \in[1, \cdots, N]$ and $\forall m \in[1, \cdots, M]$, and from these solutions the $r$ modes related to the $r$-highest eigenvalues that are expected to contain the most important information about the problem solution. For this purpose we solve the eigenvalue problem defined by Eq. (5) retaining the modes corresponding to the eigenvalues that sum up to $99 \%$ of the variance. In practice $r$ is much lower than $N$, and this constitutes the main advantage of the technique. Thus, we can try to use these $r$ eigenmodes $\phi_{k}$ for approximating the solution of a problem slightly different to the one that has served to define $u_{i}^{m}$. For this purpose we define the matrix $\boldsymbol{A}=\left[\boldsymbol{\phi}_{1} \cdots \phi_{r}\right]$ which is an orthonormal basis

$$
\boldsymbol{A}=\left(\begin{array}{llll}
\phi_{1}\left(\boldsymbol{x}_{1}\right) & \phi_{2}\left(\boldsymbol{x}_{1}\right) & \cdots & \phi_{r}\left(\boldsymbol{x}_{1}\right) \\
\phi_{1}\left(\boldsymbol{x}_{2}\right) & \phi_{2}\left(\boldsymbol{x}_{2}\right) & \cdots & \phi_{r}\left(\boldsymbol{x}_{2}\right) \\
\vdots & \vdots & \ddots & \vdots \\
\phi_{1}\left(\boldsymbol{x}_{N}\right) & \phi_{2}\left(\boldsymbol{x}_{N}\right) & \cdots & \phi_{r}\left(\boldsymbol{x}_{N}\right)
\end{array}\right)
$$

Now, assuming that the displacement vector contains the nodal degrees of freedom, it can be expressed as:

$$
\boldsymbol{u}^{m}=\sum_{i=1}^{r} \zeta_{i}^{m} \phi_{i}=\boldsymbol{A} \boldsymbol{\zeta}^{m}
$$

in which $\boldsymbol{\zeta}^{m}$ are the coordinates with respect to the POD basis. They are sometimes referred to as the reduced coordinates. It is worth reiterating that as $r$ is much smaller than $N$ the displacement vector could be expressed with a few reduced coordinates. 


\subsection{POD with Interpolation (PODI)}

In the previous section POD procedure was explained considering a timevarying field, although that procedure can be applied in parameter space for example computing displacements by varying a parameter [Ly and Tran, 2001, Epureanu et al., 2001]. In this work muscle activations were used as parameters and the displacement field was obtained for a range of physiological values. The procedure for fast prediction of the displacement field $\boldsymbol{u}$ for any value of the parameter $\gamma$ can be summarized as

1. Perform simulations for each parameter value $\gamma_{i}$ to obtain snapshots $\boldsymbol{u}^{\gamma_{i}}$.

2. Apply POD technique to obtain the matrix $\boldsymbol{A}$ in Eq. (9) which is the orthonormal basis.

3. Compute the reduced coordinates for each snapshot using the inner product as

$$
\zeta_{j}^{\gamma_{i}}=\left(\boldsymbol{u}^{\gamma_{i}}, \phi_{j}\right)
$$

4. The reduced coordinates for intermediate values of $\gamma$ that were not included in constructing POD basis are calculated by cubic spline interpolation of $\boldsymbol{\zeta}^{\gamma_{i}}$ which is the vector containing the reduced coordinates corresponding to the parameter value $\gamma_{i}$. Now, the displacement field for this parameter value can be calculated using Eq. (4) as

$$
\boldsymbol{u}^{\gamma}=\sum_{i=1}^{r} \zeta_{i}^{\gamma} \boldsymbol{\phi}_{i}=\boldsymbol{A} \boldsymbol{\zeta}^{\gamma}
$$

Remark 1. Often, one is interested in finding rather an approximating affine subspace than a linear subspace. Hence the snapshots are centered by subtracting its average, $\overline{\boldsymbol{u}}$ and collect the centered vectors as columns of the matrix $\boldsymbol{Q}$.

Remark 2. POD modes can also be computed by singular value decomposition (SVD) of $\boldsymbol{Q}\left(\boldsymbol{U} \boldsymbol{\Sigma} \boldsymbol{V}^{T}\right)$. In that case the POD modes are the columns of $\boldsymbol{U}$.

Remark 3. In many applications $N$ is very large and calculation of the POD modes would require solving a large eigenvalue problem which is not feasible. In such cases the modes can be computed by the method of snapshots proposed by [Sirovich, 1987]. 


\section{Results and discussion}

As a preliminary result, in order to investigate the ability of the model order reduction technique to run our foot model in an interactive way, one example is considered. In this example, the tibialis anterior muscle was activated and the displacement field was achieved using the Artisynth program. Afterwards the POD was applied to these solutions to construct a reduced order model with the muscle activation as a parameter. The force exerted by the tibialis anterior muscle is increased from 0 to $70 \%$ of its maximum value and the displacement field of the finite element model is computed by Artisynth and saved at each interval along with the muscle activation value (with a total of 200 simulated results). If we consider the muscle activation as a parameter we can use POD with interpolation (PODI) technique to have a fast prediction of the displacement field for any parameter value between 0 and $70 \%$. We used 101 snapshots to construct the reduced order model (ROM) and 99 snapshots to evaluate the ROM for intermediate values of muscle activation. The nodal displacements magnitude for the first three eigenmodes are shown in Fig. 6 to Fig. 8.
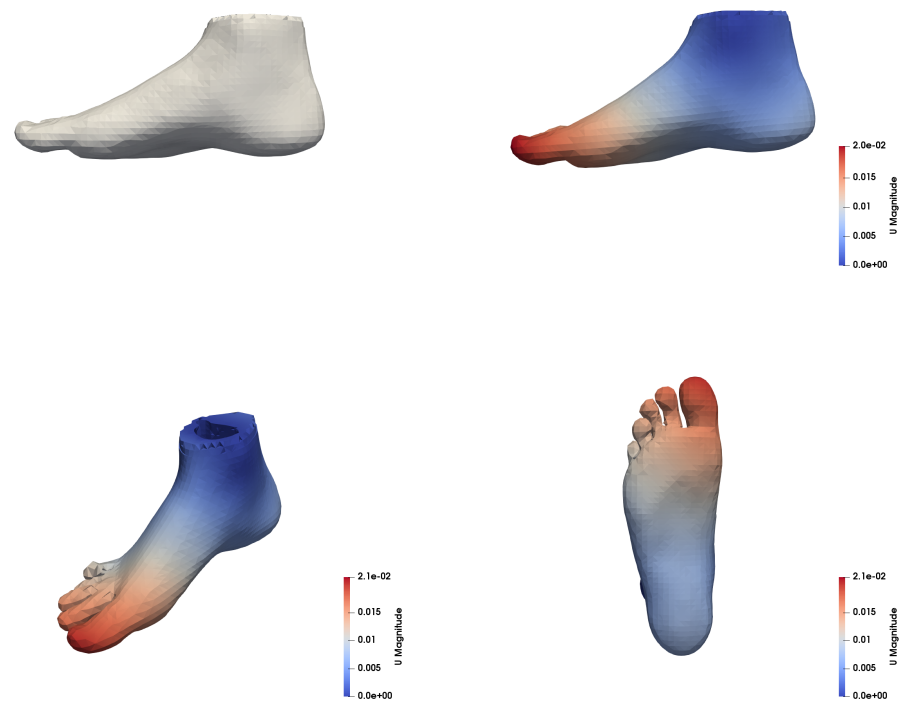

Figure 6: The nodal displacements magnitude for the first eigenmode

The displacement field due to the tibialis anterior muscle $70 \%$ activation and computed by Arisynth from one side and by the ROM from the other side are depicted in Fig. 9 and Fig. 10 respectively. 

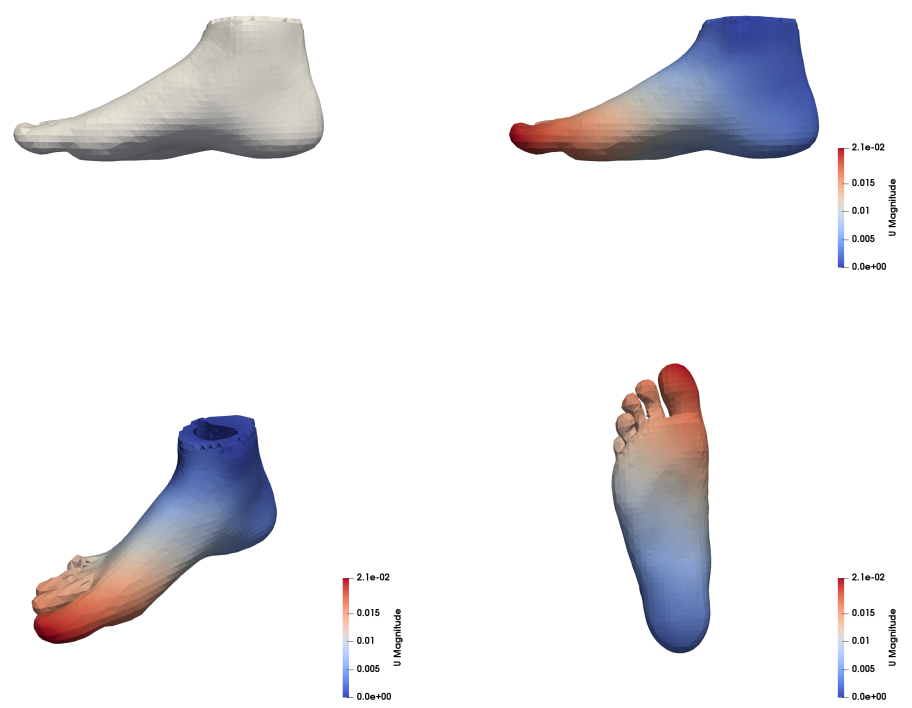

Figure 7: The nodal displacements magnitude for the second eigenmode
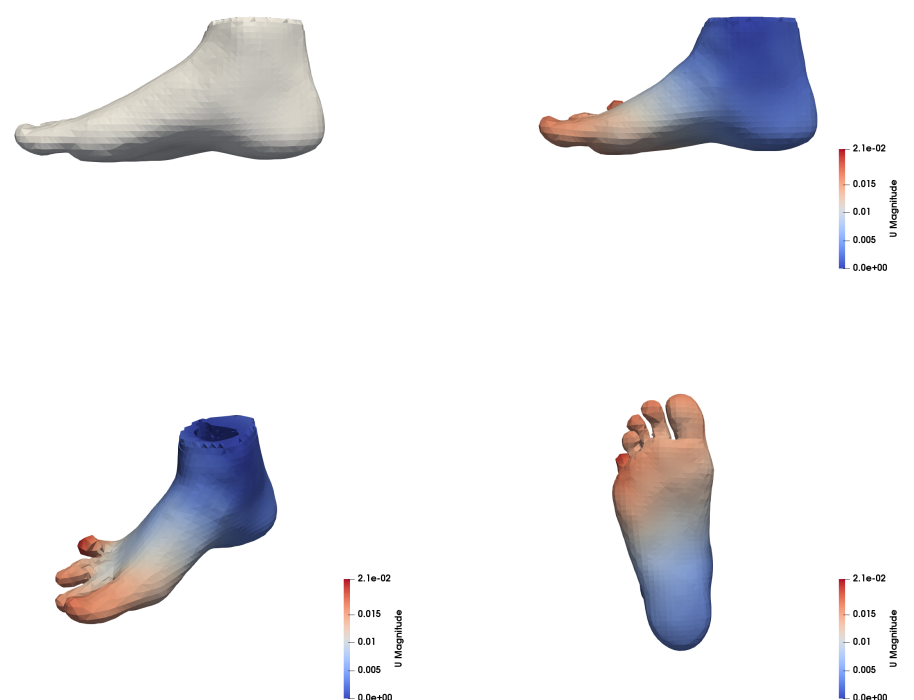

Figure 8: The nodal displacements magnitude for the third eigenmode 


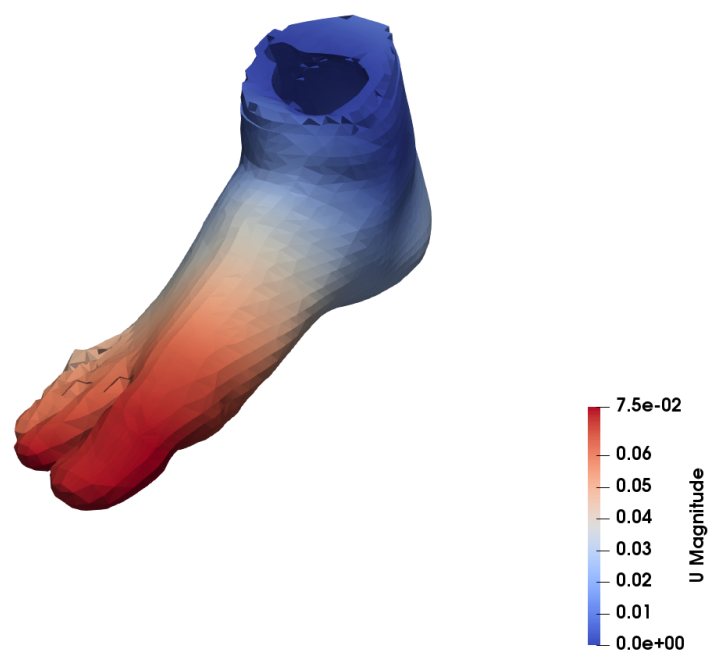

Figure 9: The displacement field computed by the Artisynth program

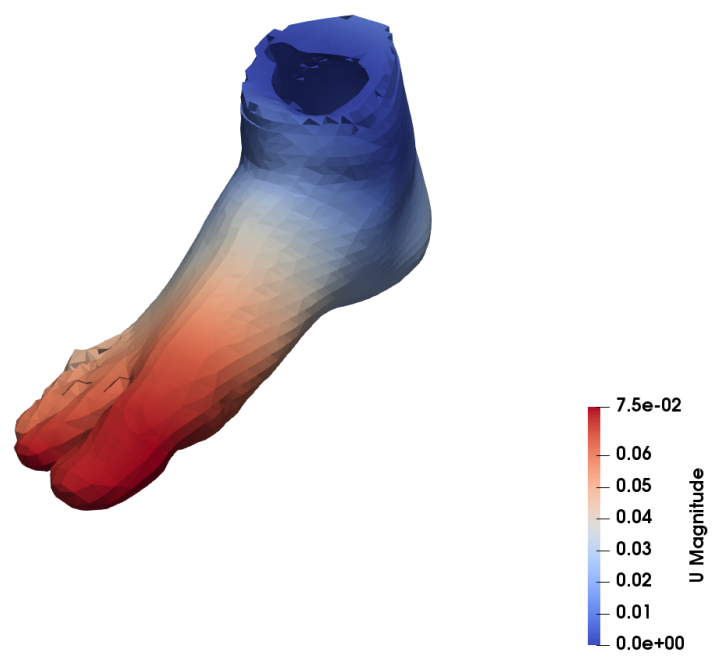

Figure 10: The displacement field computed by the reduced order model $(\mathrm{ROM})$

As it can be seen the ROM solution has a good agreement with the Artisynth solution which is considered as the reference solution. The evolution of the relative error norm $\frac{\left\|\boldsymbol{U}_{P O D I}-\boldsymbol{U}_{A r t}\right\|}{\left\|\boldsymbol{U}_{A r t}\right\|}$ with muscle activation is plotted in Fig. 11 and the error in displacement $\boldsymbol{U}_{P O D I}-\boldsymbol{U}_{A r t}$ was computed in the whole foot volume to determine the zones with less accurate results which 
are around the tip of the big toe (Fig. 12).

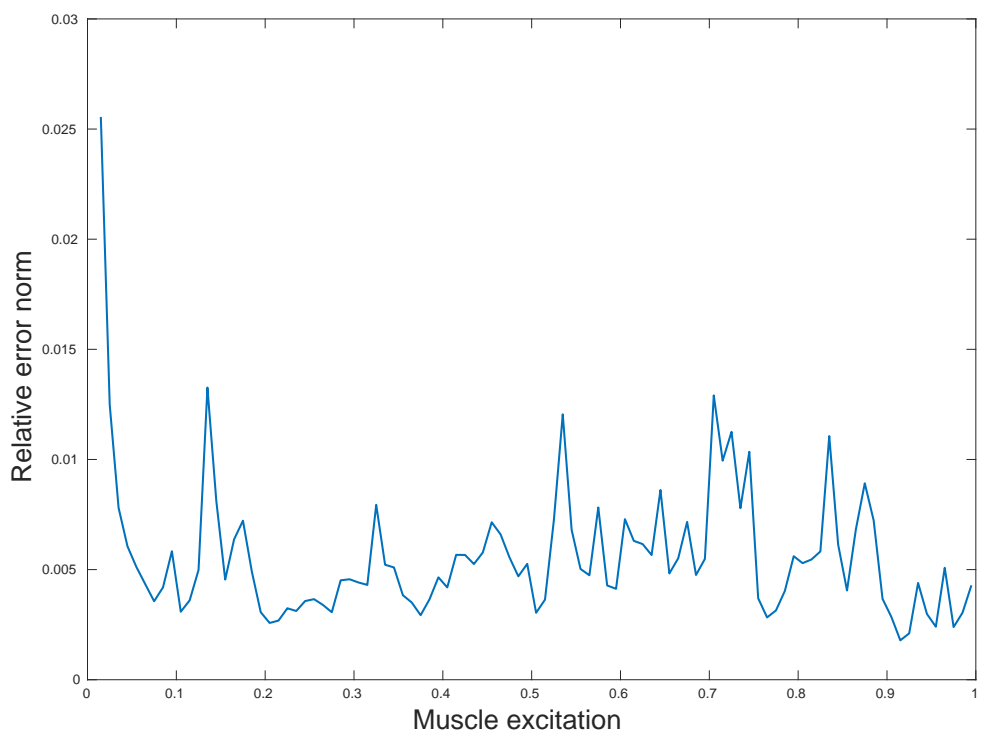

Figure 11: The relative error norm evolution with muscle activation
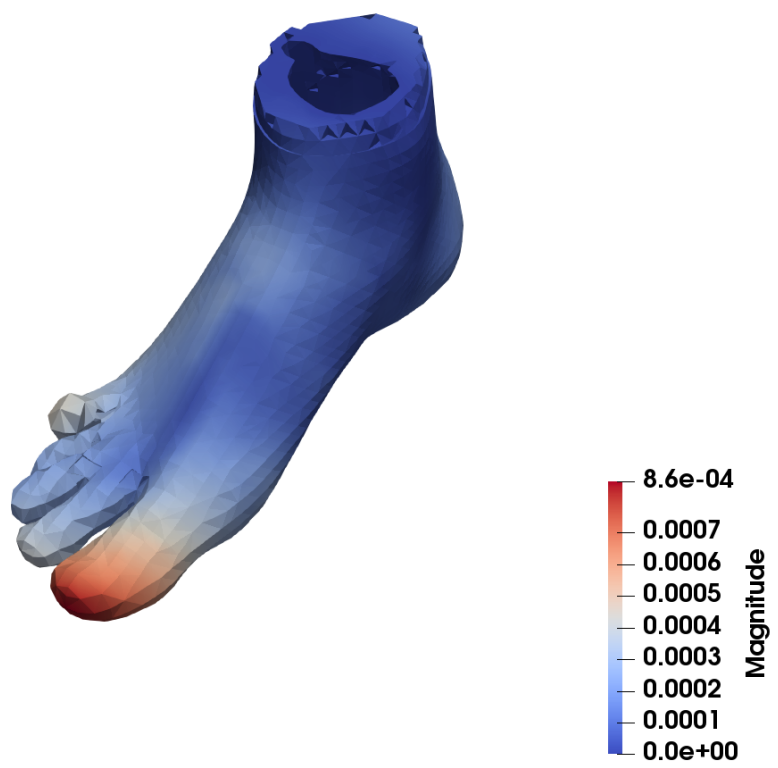

Figure 12: The error in displacement for the whole foot 
The nodes around the big toe have large displacements and the node with maximum displacement was picked. The evolution of this value with the muscle activation calculated using the Artisynth program and the ROM is plotted in Fig. 13.

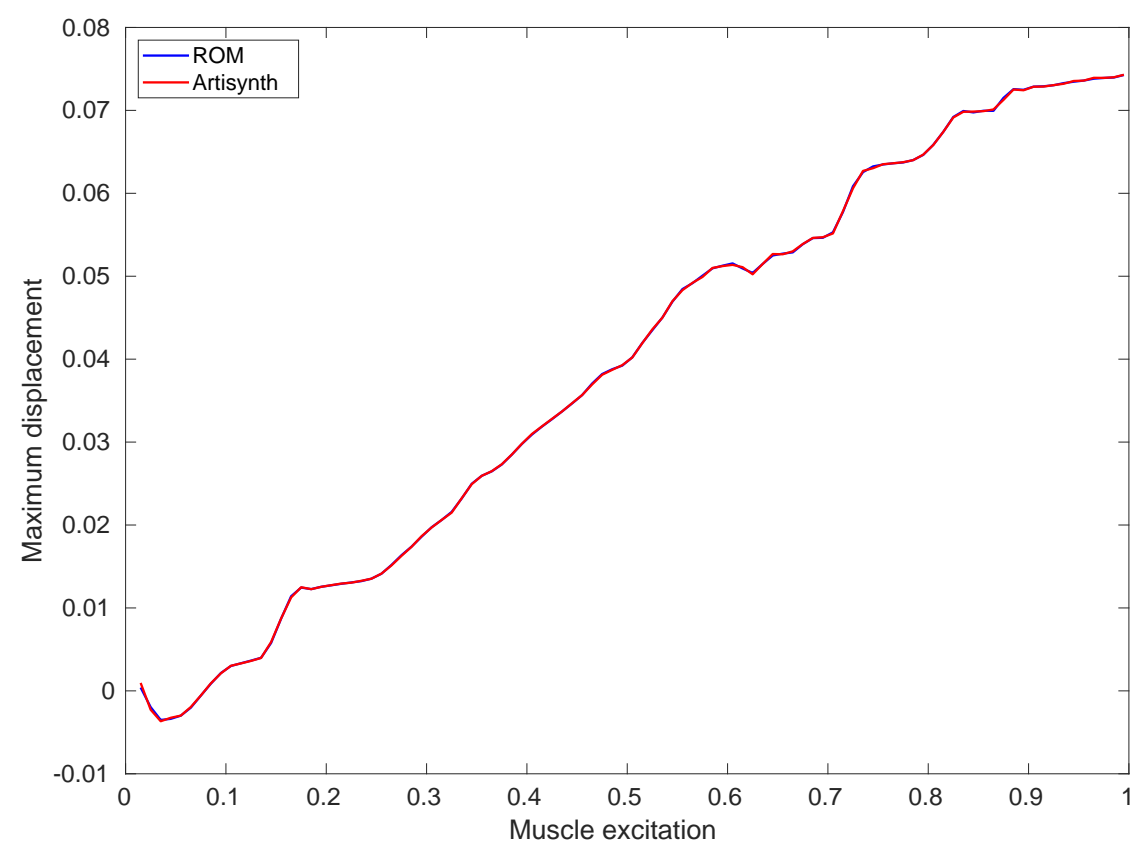

Figure 13: The maximum displacement evolution with muscle activation

The computation time using Artisynth was about 12 hours on a PC running Linux Mint with Intel ${ }^{\circledR}$ Xeon ${ }^{\circledR}$ CPU E5-1607 at $3 \mathrm{GHz}$; only four cores were used. Once the reduced order model is constructed, the displacement field generated by any activation of the tibialis anterior muscle requires 0.1 second to be computed. Despite such short computation time the results of the reduced order model show good accuracy in comparison to the Artisynth solution since the maximum relative error norm was $2.6 \%$ which seems acceptable in a clinical routine. This technique could equally be extended to use an on-line version of the foot model by applying pressure values measured by the smart sock.

\section{Acknowledgments}

This work was funded by the Fondation pour la Recherche Médicale under the project FRM DIC20161236448. 


\section{References}

Eran Atlas, Ziva Yizhar, Sam Khamis, Noa Slomka, Shlomo Hayek, and Amit Gefen. Utilization of the foot load monitor for evaluating deep plantar tissue stresses in patients with diabetes: Proof-of-concept studies. Gait and Posture, 29(3):377 - 382, 2009. ISSN 0966-6362.

Andrew JM Boulton, Loretta Vileikyte, Gunnel Ragnarson-Tennvall, and Jan Apelqvist. The global burden of diabetic foot disease. Lancet, 366: 1719-1724, 2005.

Elías Cueto and Francisco Chinesta. Real time simulation for computational surgery: a review. Advanced Modeling and Simulation in Engineering Sciences, 1(1):11, Apr 2014. ISSN 2213-7467. doi: 10.1186/2213-7467-1-11.

P. de Leva. Adjustments to zatsiorsky-seluyanovs segment inertia parameters. Journal of biomechanics, 29(9):1223-30, 1996.

B. I. Epureanu, E. H. Dowell, and K.C. Hall. A parametric analysis of reduced order models of potential flows in turbomachinery using proper orthogonal decomposition. In ASME TURBO EXPO 2001, 2001.

Amit Gefen. Plantar soft tissue loading under the medial metatarsals in the standing diabetic foot. Medical Engineering and Physics, 25(6):491 - 499, 2003. ISSN 1350-4533.

K. Karhunen. Uber lineare methoden in der wahrscheinlichkeitsrechnung. Ann. Acad. Sci. Fennicae, ser. Al. Math. Phys., 37, 1946.

P. Krysl, S. Lall, and J. E. Marsden. Dimensional model reduction in non-linear finite element dynamics of solids and structures. International Journal for Numerical Methods in Engineering, 51(4):479-504. doi: 10.1002/nme.167.

Y.C. Liang, H.P. Lee, S.P. Lim, W.Z. Lin, K.H. Lee, and C.G. Wu. Proper orthogonal decomposition and its applications-part I: Theory. Journal of Sound and Vibration, 252(3):527 - 544, 2002. ISSN 0022-460X. doi: https://doi.org/10.1006/jsvi.2001.4041.

E. Linder-Ganz, N. Shabshin, Y. Itzchak, Z. Yizhar, I. Siev-Ner, and A. Gefen. Strains and stresses in sub-dermal tissues of the buttocks are greater in paraplegics than in healthy during sitting. Journal of Biomechanics, 41(3):567-80, 2008. 
John E. Lloyd, Ian Stavness, and Sidney Fels. Artisynth: A fast interactive biomechanical modeling toolkit combining multibody and finite element simulation. In Yohan Payan, editor, Soft Tissue Biomechanical Modeling for Computer Assisted Surgery, pages 355-394. Springer Berlin Heidelberg, 2012.

S. Loerakker, E. Manders, G. J. Strijkers, K. Nicolay, F. P. T. Baaijens, D. L. Bader, and C. W. J. Oomens. The effects of deformation, ischemia, and reperfusion on the development of muscle damage during prolonged loading. Journal of Applied Physiology, 111(4):1168-1177, 2011. doi: 10.1152/japplphysiol.00389.2011. PMID: 21757578.

V. Luboz, A. Perrier, M. Bucki, B. Diot, F. Cannard, N. Vuillerme, and Y. Payan. Influence of the calcaneus shape on the risk of posterior heel ulcer using 3d patient-specific biomechanical modeling. Annals of Biomedical Engineering, 43(2):325-335, Feb 2015. ISSN 1573-9686. doi: 10.1007/s10439-014-1182-6.

Hung V. Ly and Hien T. Tran. Modeling and control of physical processes using proper orthogonal decomposition. Mathematical and Computer Modelling, 33(1):223 - 236, 2001. ISSN 0895-7177. doi: https://doi.org/10.1016/S0895-7177(00)00240-5. Computation and control VI proceedings of the sixth Bozeman conference.

S. Niroomandi, I. Alfaro, E. Cueto, and F. Chinesta. Real-time deformable models of non-linear tissues by model reduction techniques. Computer Methods and Programs in Biomedicine, 91(3):223 - 231, 2008. ISSN 01692607. doi: DOI: 10.1016/j.cmpb.2008.04.008.

S. Niroomandi, I. Alfaro, E. Cueto, and F. Chinesta. Accounting for large deformations in real-time simulations of soft tissues based on reduced-order models. Computer Methods and Programs in Biomedicine, In Press, Corrected Proof:-, 2010. ISSN 0169-2607. doi: DOI: 10.1016/j.cmpb.2010.06.012.

A. Perrier, N. Vuillerme, V. Luboz, M. Bucki, F. Cannard, B. Diot, D. Colin, D. Rin, J.-P. Bourg, and Y. Payan. Smart Diabetic Socks: Embedded device for diabetic foot prevention. Innovation and Research in BioMedical engineering, 35:72-76, April 2014.

Antoine Perrier, Marek Bucki, Vincent Luboz, Nicolas Vuillerme, and Yohan Payan. 3D musculoskeletal finite element analysis of the foot kinematics under muscle activation with and without ankle arthrodesis. Computer 
Methods in Biomechanics and Biomedical Engineering, 0(0):1-2, 2015. doi: 10.1080/10255842.2015.1069605. PMID: 26273957.

Antoine Perrier, Vincent Luboz, Marek Bucki, Francis Cannard, Nicolas Vuillerme, and Yohan Payan. Biomechanical modeling of the foot. In Biomechanics of Living Organs: Hyperelastic Constitutive Laws for Finite Element Modeling, pages 545-563. Academic Press, 2017.

René Pinnau. Model reduction via proper orthogonal decomposition. In Model Order Reduction: Theory, Research Aspects and Applications, volume 13, pages 95-109. Springer-Verlag Berlin Heidelberg, 2008.

Jonathan E Shaw and Andrew JM Boulton. The pathogenesis of diabetic foot problems: An overview. Diabetes, 46(Supplement 2):S58-S61, 1997. ISSN 0012-1797. doi: 10.2337/diab.46.2.S58.

Lawrence Sirovich. Turbulence and the dynamics of coherent structures. i iii. Quarterly of Applied Mathematics, 45(3):561-1590, 1987. 